\title{
Higher-Order Theory for Functionally Graded Materials
}
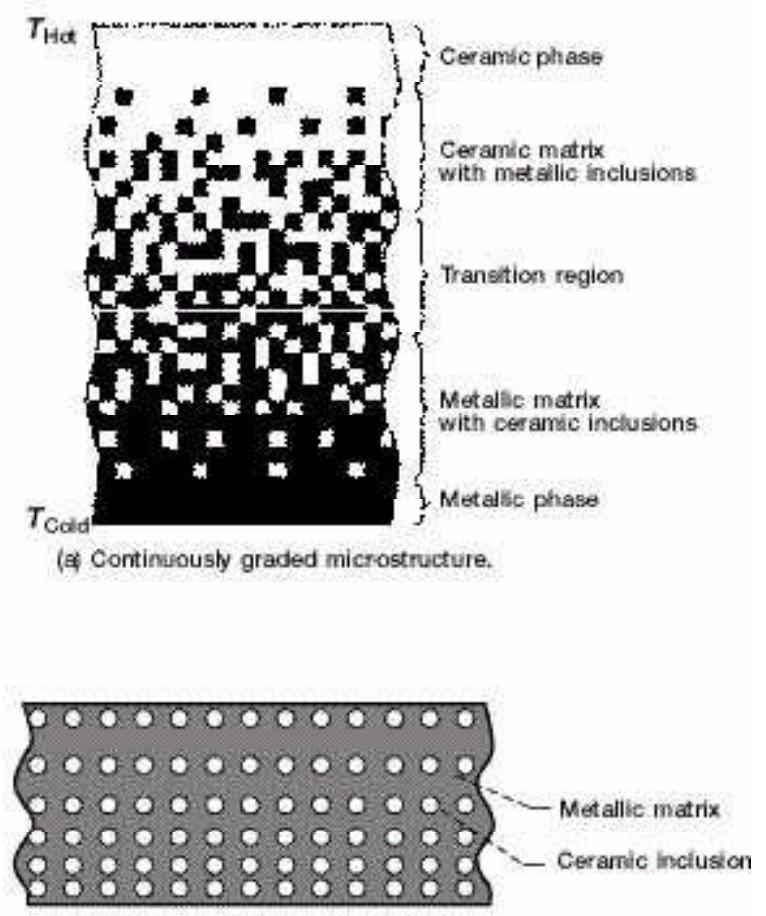

(b) Discretely graded microstructure.

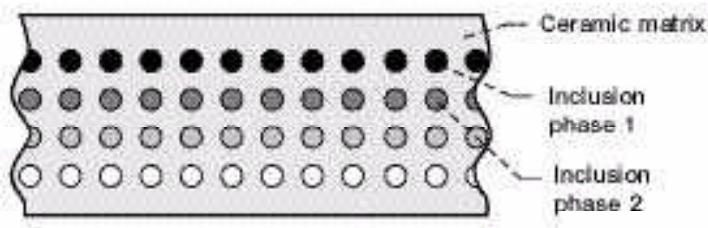

(c) Mutiphase gradied microstructure.

\section{Examples of different types of functionally graded microstructures.}

Functionally graded materials (FGM's) are a new generation of engineered materials wherein the microstructural details are spatially varied through nonuniform distribution of the reinforcement phase(s), see the top figure. Engineers accomplish this by using reinforcements with different properties, sizes, and shapes, as well as by interchanging the roles of the reinforcement and matrix phases in a continuous manner (ref. 1). The result is a microstructure that produces continuously or discretely changing thermal and mechanical properties at the macroscopic or continuum scale. This new concept of engineering the material's microstructure marks the beginning of a revolution both in the materials science and mechanics of materials areas since it allows one, for the first time, to fully integrate the material and structural considerations into the final design of structural components. Functionally graded materials are ideal candidates for applications involving severe thermal gradients, ranging from thermal structures in advanced aircraft and 
aerospace engines to computer circuit boards. Owing to the many variables that control the design of functionally graded microstructures, full exploitation of the FGM's potential requires the development of appropriate modeling strategies for their response to combined thermomechanical loads. Previously, most computational strategies for the response of FGM's did not explicitly couple the material's heterogeneous microstructure with the structural global analysis. Rather, local effective or macroscopic properties at a given point within the FGM were first obtained through homogenization based on a chosen micromechanics scheme and then subsequently used in a global thermomechanical analysis.

Reference 2 provides a detailed review and description of the full generalization of a new Cartesian-coordinate-based higher order theory for functionally graded materials developed by the University of Virginia in conjunction with the NASA Glenn Research Center over the past several years. This theory circumvents the problematic use of the standard micromechanical approach, based on the concept of a representative volume element, commonly employed in the analysis of functionally graded composites by explicitly coupling the local (microstructural) and global (macrostructural) responses. The theoretical framework is based on volumetric averaging of the various field quantities, together with the imposition of boundary and interfacial conditions in an average sense between the subvolumes used to characterize the composite's functionally graded microstructure. The generalization outlined involved the extension of the theoretical framework to enable the analysis of materials characterized by spatially variable microstructures in all three directions. Specialization of the generalized theoretical framework to previously published versions of the higher order theory for materials functionally graded in one and two directions was demonstrated. Reference 2 summarizes the major findings obtained with the one- and two-directional versions of the higher order theory. The results illustrate both the fundamental issues related to the influence of microstructure on microscopic and macroscopic quantities governing the response of composites and technologically important applications. A major issue addressed was the applicability of the classical homogenization schemes in the analysis of functionally graded materials. The technologically important applications illustrate the utility of functionally graded microstructures in tailoring the response of structural components in a variety of applications involving uniform and gradient thermomechanical loading.

\section{References}

1. Hirai, T.: Functional Gradient Materials. Processing of Ceramics, pt. 2, vol. 17B, Richard J. Brook, et al., eds., Weinheim, New York, NY, 1996, pp. 293-341.

2. Aboudi, J.; Pindera, M.J.; and Arnold, S.M.: Higher-Order Theory for Functionally Graded Materials. Composites Part B--Engineering, vol. 30, no. 8, 1999, pp. 777832.

Glenn contact: Dr. Steven M. Arnold, 216-433-3334, Steven.M.Arnold@ grc.nasa.gov Authors: Prof. J. Aboudi, Prof. M.J. Pindera, and Dr. Steven M. Arnold Headquarters program office: OAT 
Programs/Projects: HOTPC

Special recognition: Life Prediction Best Branch Paper 2000 